\title{
Candidíase oral em usuários de próteses dentárias removíveis: fatores associados
}

\author{
Oral candidiasis in users of removable dental prostheses: associated factors \\ Método de adherencia al tratamiento en pacientes hipertensos
}

\author{
Julliana Cariry Palhano FREIRE ${ }^{1}$ \\ Marina Tavares Costa NÓBREGA ${ }^{\mathbf{1}}$ \\ Stéphanie Cariry Palhano FREIRE ${ }^{2}$ \\ Eduardo DIAS-RIBEIRO ${ }^{3}$
}

${ }^{1}$ Cirurgiã-Dentista, Mestranda em Ciências Odontológicas, Universidade Federal da Paraíba - UFPB, 58051 - 900 João Pessoa - PB, Brasil

${ }^{2}$ Enfermeira. Hospital Universitário Alcides Carneiro - HUACUFCG, 58107670 Campina Grande - PB, Brasil

${ }^{3}$ Doutor. Universidade Federal de Campina Grande - UFCG, 58429-900 Patos - PB, Brasil

\begin{abstract}
Resumo
A estomatite protética tem sido considerada a lesão bucal mais frequentemente observada em usuários de próteses removíveis. Em portadores de próteses está comumente associada à presença de Candida. Esse estudo objetivou realizar um levantamento da literatura sobre os fatores associados ao desenvolvimento de candidíase oral em usuários de próteses dentárias removíveis. Realizou-se uma busca na literatura através da base de dados PUBMED com as seguintes palavras-chave: Candida, Candidíase bucal e Prótese dentária. Foram encontrados 12 artigos científicos que se enquadraram ao tema proposto. Diante dos resultados, observou-se que a estomatite protética está relacionada à presença de Candida. Em usuários de próteses, essa presença de espécies de Candida é maior do que em não usuários e a espécie mais isolada é a C. albicans. A má higienização da prótese é um dos fatores associados ao desenvolvimento de candidíase oral. Cuidados com a higiene oral e da prótese são necessários para o controle da microbiota oral e prevenção da candidíase oral.
\end{abstract}

Descritores: Candida; Candidíase Bucal; Prótese Dentária.

\begin{abstract}
Prosthetic stomatitis has been considered the most frequently observed oral lesion in users of removable prostheses. In patients with prostheses, it is commonly associated with the presence of Candida. This study aimed to perform a literature review on the factors associated with the development of oral candidiasis in users of removable dental prostheses. A literature search was performed through the PUBMED database with the following keywords: Candida, Oral Candidiasis and Dental Prosthesis. Twelve scientific articles were found that fit the proposed theme. In view of the results, it was observed that prosthetic stomatitis is related to the presence of Candida. In prosthesis users, this presence of Candida species is higher than in non-users and the most isolated species is C. albicans. Poor hygiene of the prosthesis is one of the factors associated with the development of oral candidiasis. Oral hygiene and prosthesis care are necessary to control the oral microbiota and prevent oral candidiasis.

Descriptors: Candida; Candidiasis, Oral; Dental Prosthesis.
\end{abstract}

\begin{abstract}
Resumen
Estomatitis protésica se ha considerado que las lesiones orales se presentan con más frecuencia en los usuarios de dentaduras postizas. En los pacientes con prótesis se asocia comúnmente con la presencia de Candida. Este estudio tuvo como objetivo realizar un estudio de la literatura sobre los factores asociados con el desarrollo de la candidiasis oral en los usuarios de dentaduras postizas. Se realizó una búsqueda bibliográfica a través de la base de datos PubMed con las siguientes palabras clave: Candida, la candidiasis oral y prótesis dental. Encontraron 12 artículos que cumplían con el tema propuesto. De los resultados, se observó que la estomatitis protésica está vinculada a la presencia de Candida. En los usuarios de dentaduras postizas, que la presencia de especies de Candida es mayor que en las no usuarias y las especies más aisladas es C. albicans. La falta de higiene de la prótesis es uno de los factores asociados con el desarrollo de candidiasis oral. Se necesitan ayudas para el cuidado y la higiene oral para controlar la microflora bucal y la prevención de la candidiasis oral.

Descriptores: Candida; Candidiasis Bucal; Prótesis Dental.
\end{abstract}

\section{INTRODUÇÃO}

A candidíase consiste em uma extensa variedade de síndromes clínicas causadas por um fungo do gênero Candida $^{I}$. Entre os fatores predisponentes para a candidíase, têm-se: próteses intraorais, deficiências nutricionais, doenças metabólicas, drogas antibacterianas, deficiência orgânica das glândulas salivares e imunossupressão ${ }^{2}$.

A estomatite protética tem sido considerada a lesão bucal mais frequentemente observada em usuários de próteses removíveis, apresentando uma prevalência de 60 a $72 \%{ }^{3}$. Em pacientes portadores de aparelhos protéticos, é comum o surgimento de estomatite protética quase sempre associada à presença de candidíase eritematosa ${ }^{4}$.

A presença de dispositivos orais como próteses e aparelhos ortodônticos podem alterar o ambiente oral, levando a modificações nas características físicas e biológicas da saliva e outras estruturas orais, muitas vezes causando um desequilíbrio na microbiota local ${ }^{5}$.

A prótese pode interferir ou contribuir para a detecção de processos clínicos ou subclínicos resultantes da interação entre a prótese e os microrganismos. Além disso, a prótese é 
muitas vezes relacionada com o aparecimento de lesões orais, tais como úlceras traumáticas, hiperplasias mucogengivais e candidíase oral ${ }^{6}$. A higienização e a desinfecção diárias de próteses dentárias removíveis são necessárias para promover a saúde e a conservação dos tecidos orais. A manutenção da mucosa saudável está relacionada ao grau de limpeza das próteses em contato com tecidos orais ${ }^{7}$.

Diante do exposto, o presente estudo objetivou realizar um levantamento da literatura sobre os fatores associados ao desenvolvimento de candidíase oral em usuários de próteses dentárias removíveis.

\section{MATERIAL E MÉTODO}

Realizou-se busca na literatura através da base de dados PUBMED com os seguintes descritores: Candida, Candidíase Bucal e Prótese Dentária. Foram selecionados os estudos relacionados ao tema dessa pesquisa, sem obstrução de tempo.

\section{RESULTADOS E DISCUSSÃO}

No estudo de Bianchi et al. $^{7}$ os pacientes que utilizaram próteses dentárias removíveis foram 6,9 vezes mais propensos a desenvolver candidíase oral do que idosos não portadores. Este fato pode ser explicado pelas condições precárias das próteses dentárias (má adaptação e fabricação) ou alta porosidade devido ao uso prolongado. A inadequada higienização da prótese realizada pela maioria dos pacientes que utilizam próteses dentárias removíveis é um fator relacionado ao aumento do número de Candida spp. $\mathrm{O}$ agente etiológico mais comum isolado na pesquisa desses autores foi a $C$. albicans. Os autores concluem que a utilização de próteses e pobre higiene oral em pacientes idosos são fatores predisponentes ao desenvolvimento da candidíase oral.

Prakash et al. ${ }^{8}$ verificaram que a prevalência de diferentes espécies de Candida foi significativamente maior em usuários de próteses sendo as espécies mais predominantes: $C$. albicans, $C$. tropicalis, $C$. dubliensis e $C$. glabrata. A idade foi um fator relacionado à prevalência de Candida.

Marinoski et al. ${ }^{9}$ concluíram que a pobre higiene oral e da prótese, seu uso noturno e a mucosa com $\mathrm{pH}$ inferior a 6,5 são fatores etiológicos locais predominantes que contribuem para o desenvolvimento de estomatite protética. Nenhuma associação significativa foi encontrada entre estomatite protética e achados microbiológicos, idade ou tipo das próteses, presença de prótese anterior e hábito de fumar. Patil et al. ${ }^{10}$ encontraram relação estatisticamente significante entre uso de prótese e candidíase.

Altarawneh et al. ${ }^{11}$ concluem que o fator etiológico importante para estomatite protética na população de pacientes edêntulos é a presença de Candida em prótese e saliva. Verificou-se que outros fatores, tais como o fluxo salivar, xerostomia, adaptação da prótese e a presença de Candida na mucosa, são menos importantes nesta população.

Witzel et al. ${ }^{12}$ relatam que o uso de próteses parciais removíveis foi um fator importante para o isolamento de $C$. albicans em pacientes $\mathrm{HIV}^{+}$, e o nível de CD4 parece desempenhar um papel na presença de candidíase oral, pois a contagem de células CD4 foi menor em pacientes com candidíase oral independente do uso de próteses removíveis.

Em uma revisão sistemática da literatura Emami et al. ${ }^{13}$ encontraram associação entre a estomatite protética e o uso de próteses parciais removíveis. No entanto, devido a limitações metodológicas e desenhos transversais de estudos de pesquisa, não pode ser inferida relação de causaefeito. Ainda afirmam que pesquisas futuras devem fornecer maiores níveis de evidência para confirmar a etiologia da estomatite protética em usuários próteses parciais removíveis.

Numa revisão de literatura, Gendreau et al. ${ }^{14}$ consideram que fatores etiológicos incluem má higiene da prótese, seu uso continuo e noturno desgastando as próteses removíveis, acúmulo de placa na prótese, e contaminação por bactérias e leveduras da superfície da prótese. Todos estes fatores parecem aumentar a capacidade de $C$. albicans, tanto para colonizar a prótese e superfícies das mucosas orais, onde atua como um agente patogénico oportunista.

Zomorodian et al. ${ }^{15}$ demonstraram uma associação significativa entre a duração e desgaste da prótese com a candidíase oral. Além disso, observou-se uma alta prevalência de $C$. dubliniensis em usuários de próteses.

Segundo Abaci et al. ${ }^{16} C$. albicans vivem como saprófitas na cavidade oral. No entanto, é capaz de causar infecção se houver condições predisponentes relacionadas ao hospedeiro. O uso de prótese removível pode fazer com que esses microrganismos ganhem patogenicidade. Em seu estudo, a espécie mais frequentemente encontrada foi a $C$. albicans e a higienização da prótese foi relacionada ao aumento do número de Candida e ao desenvolvimento de estomatite protética.

Gasparoto et al. ${ }^{17}$ detectaram a $C$. dubliniensis no ambiente bucal de portadores de próteses. A associação de C. dubliniensis com C. albicans ocorreu em aproximadamente $10 \%$ dos casos de estomatite protética. Daniluk et al. ${ }^{18}$ relatam que a taxa de ocorrência de $C$. albicans em sua pesquisa foi maior em pacientes usuários de próteses.

\section{CONCLUSÃO}

A presença de Candida em usuários de próteses é maior do que entre os não usuários, sendo a $C$. albicans a mais frequentemente isolada. A má higienização da prótese, sendo esta um fator retentivo de biofilme, está associada ao desenvolvimento da candidíase oral.

\section{REFERÊNCIAS}

1. Álvares CA, Svidzinski TIE, Consolaro MEL. Candidíase vulvuvaginal: fatores predisponentes do hospedeiro e virulência das leveduras. J Bras Patol Med Lab. 2007; 43(5):319-27.

2. Kleinegger C, Stoeckel D, Kurago Z. A comparison of salivary calprotectin levels in subjects with and without oral candidiasis. Oral Surg Oral Pathol Oral Radiol Endod. 2001; 92(1): 62-7.

3. Webb BC, Thomas CJ, Whittle T. A 2-year study of Candida-associated denture stomatitis treatment in aged care subjects. Gerodontology. 2005; 22(3):168-76.

4. Batista JM, Birman EG, Cury AE. Suscetibilidade a antifúngicos de cepas de Candida albicans isoladas de pacientes com estomatite protética. Rev Odontol Univ São Paulo. 1999; 13(4):343-8.

5. Campbell CHCT. Alterações da microflora bucal em pacientes portadores de aparelho ortodôntico fixo. Ortodon Gaúcha. 2003; 7(2): 98-109.

6. Goiato MC, Castelleoni L, Santos DM, Gennari H, Filho, Assunção WG. Lesões orais provocadas pelo uso de próteses removíveis. Pesq Bras Odontoped Clin Integr. 2005; 5:85-90.

7. Bianchi CMP de C, Bianchi HA, Tadano T, et al. Factors related to oral candidiasis in elderly users and 
non-users of removable dental prostheses. Rev Inst Med trop S Paulo. 2016; 58:17.

8. Prakash B, Shekar M, Maiti B, Karunasagar I, Padiyath S. Prevalence of Candida spp. among healthy denture and nondenture wearers with respect to hygiene and age. J Ind Prosthodontic Soc. 2015; 15(1):29-32.

9. Marinoski J, Bokor-Bratić M, Čanković M. Is denture stomatitis always related with candida infection? A case control study. Med Glas (Zenica). 2014; 11(2): 379-84.

10. Patil S, Yadav N, Patil P, Kaswan S. Prevalence and the relationship of oral mucosal lesions in tobacco users and denture wearers in the North Indian population. J Family Community Med. 2013; 20(3):187-191.

11. Altarawneh S, Bencharit S, Mendoza L, et al. Clinical and histological findings of denture stomatitis as related to intraoral colonization patterns of $C$. albicans, salivary flow, and dry mouth. J Prosthodont. 2013; 22(1):13-22.

12. Witzel AL, PiresM de F, de Carli ML, Rabelo GD, Nunes TB, da Silveira FR. Candida albicans isolation from buccal mucosa of patients with HIV wearing removable dental prostheses. Int $\mathrm{J}$ Prosthodont. 2012; 25(2):127-31.

13. Emami E, Taraf H, de Grandmont P, Gauthier G, de Koninck L, Lamarche C, de Souza RF. The association of denture stomatitis and partial removable dental prostheses: a systematic review. Int J Prosthodont. 2012; 25(2):113-9.

14. Gendreau L, Loewy ZG. Epidemiology and Etiology of Denture Stomatitis. J Prosthodont; 20(4):251-60.

15. Zomorodian K, Haghighi NN, Rajaee N, Pakshir K, Tarazooie B, Vojdani M, et al. Assessment of Candida species colonization and denture-related stomatitis in complete denture wearers. Med Mycol. 2011; 49(2): 208-11.

16. Abaci O, Haliki-Uztan A, Ozturk B, Toksavul S, Ulusoy M, Boyacioglu H. Determining Candida spp. incidence in denture wearers. Mycopathologia. 2010; 169(5):36572 .

17. Gasparoto TH, Dionísio TJ, de Oliveira CE, Porto VC, Gelani V, Santos CF, et al.Isolation of Candida dubliniensis from denture wearers. J Med Microbiol. 2009; 58(Pt 7):959-62.

18. Daniluk T, Tokajuk G, Stokowska W, Fiedoruk K, Sciepuk M, Zaremba ML, et al. Occurrence rate of oral Candida albicans in denture wearer patients. Adv Med Sci. 2006; 51(Suppl 1):77-80.

\section{CONFLITO DE INTERESSES}

Os autores declaram não haver conflitos de interesse.

\section{AUTOR PARA CORRESPONDÊNCIA}

Julliana Cariry Palhano Freire

jullianapalhano@hotmail.com

Submetido em 25/01/2017 Aceito em 01/03/2017 